\title{
Monocyte chemoattractant protein 1 (MCP-1) released from Helicobacter pylori stimulated gastric epithelial cells induces cyclooxygenase 2 expression and activation in T cells
}

\author{
S Futagami, T Hiratsuka, A Tatsuguchi, K Suzuki, M Kusunoki, Y Shinii, K Shinoki, \\ T lizumi, T Akamatsu, H Nishigaki, K Wada, K Miyake, K Gudis, T Tsukui, C Sakamoto
}

See end of article for authors' affiliations

Correspondence to: Dr C Sakamoto, Third Department of Internal Medicine, Nippon Medica School, 1-1-5, Sendagi,

Bunkyo-ku, Tokyo

113-8603, Japan

choitsu@nms.ac.jp

Accepted for publication 2 April 2003

\begin{abstract}
Background and aims: To clarify the interaction between gastric epithelial and mucosal T cells, we examined the role of cytokines released from epithelial cells in response to Helicobacter pylori water extract protein (HPWEP) in regulating T cell cyclooxygenase 2 (COX-2) expression and activation. Methods: Media from MKN-28 cells incubated with HPWEP for 48 hours were added to Jurkat T cells and human peripheral T cells. C-C and CXC chemokine concentrations in MKN-28 cell media, and COX-2 expression, interferon $\gamma$ (IFN- $\gamma$ ), and interleukin (IL)- 4 secretions in T cells were determined by western blot analysis and ELISA methods. Distributions of COX-2 positive T cells and monocyte chemoattractant protein 1 (MCP-1) in tissue specimens with $\mathrm{H}$ pylori associated gastritis were determined as single or double labelling by immunohistochemistry.

Results: MCP-1, IL-7, IL-8, and RANTES were detected in media from MKN- 28 cells incubated with HPWEP. Media as a whole, and MCP-1 alone, stimulated COX-2 expression and peripheral T cell proliferation. Anti-MCP-1 antibody inhibited media stimulated COX-2 mRNA expression in Jurkat T cells. Media stimulated IFN- $\gamma$ but not IL-4 secretion from peripheral T cells, while MCP- 1 stimulated IL-4 but not IFN- $\gamma$ secretion. Both stimulated cytokine release, and peripheral T cell proliferation was partially inhibited by NS-398, a specific COX-2 inhibitor. In mucosa with gastritis, COX-2 was expressed in T cells and MCP-1 was localised mainly in epithelial and mononuclear cells. MCP- 1 levels and the intensity of COX-2 expression in tissue samples were closely related.

Conclusions: Cytokines such as MCP-1, released from gastric epithelial cells in response to HPWEP, seem to modulate $T$ cell immune responses, at least in part via COX-2 expression.
\end{abstract}

Gastric and colonic epithelial cells are involved in immunological and inflammatory processes, serving not only as a surface for nutrient absorption but also as a defence against ingested pathogens, ${ }^{12}$ and express and generate soluble inflammatory mediators. In Helicobacter pylori associated gastritis, mucosal concentrations of cytokines such as interleukin (IL)-1 $\beta$, interferon (IFN)- $\gamma$, and IL-8 are significantly elevated compared with those in normal mucosa. ${ }^{34}$ In response to $H$ pylori, IL-8 secreted from gastric epithelial cells has been shown to induce neutrophil accumulation, leading to local inflammation in the gastric mucosa. However, it has yet to be conclusively clarified how infiltration of mononuclear cells such as lymphocytes and macrophages observed in chronic gastritis is regulated in the gastric mucosa, as has been shown in IL-8 neutrophil regulation.

Prostaglandins (PGs), synthesised and secreted by most human tissues and cell types, play key roles in the regulation of humoral immunity and local cell mediated immunity, modulating cytokine and Ig production as well as T cell proliferation and activation. ${ }^{56}$ Cyclooxygenases (COX) catalyse a two step conversion of arachidonic acid to $\mathrm{PGH}_{2}$, the first reaction required for biosynthesis of various PGs. COX-2 is an inducible enzyme whose induction and expression is dynamically regulated by growth factors, mitogens, tumour growth promoters, and physiological stresses. ${ }^{78}$ Persistent activation of COX-2 is associated with oncogenesis as well as with increased invasive potential of tumour cells. ${ }^{90}$ Our previous studies have suggested that COX-2 expressed in macrophages and mononuclear cells might play an important role in the mucosal repair mechanism in experimental ulcer bearing ani- mals, and in ulceration caused by $H$ pylori in humans. ${ }^{11-13}$ In addition, some reports have shown that COX-2 induced in $\mathrm{T}$ cells may regulate $\mathrm{T}$ cell cytokine release, thereby modulating immune responses. ${ }^{14}$ Studies have also shown that $\mathrm{T}$ cell polarisation may be a key factor determining whether gastritis worsens or resolves. ${ }^{15}{ }^{16}$ In coeliac disease, COX-2 expressed in $\mathrm{T}$ cells of the small intestine has been suggested to contribute to healing of the diseased mucosa. ${ }^{17}$ However, whether T cells express COX-2 in $H$ pylori gastritis mucosa or whether $\mathrm{T}$ cell activation and polarisation are related to its COX-2 expression is yet to be determined. In addition, it remains to be seen whether soluble factors released from gastric epithelial cells in response to $H$ pylori are involved in COX-2 expression and gastric mucosal $\mathrm{T}$ cell activation. In the present study, we therefore investigated the interaction between gastric epithelial cells and $\mathrm{T}$ cells by examining the role of cytokines released from gastric epithelial cells in response to $H$ pylori water extract protein with regard to T cell COX-2 expression and $\mathrm{T}$ cell activation.

Abbreviations: MCP-1, monocyte chemoattractant protein 1; COX, cyclooxygenase; IL, interleukin; IFN- $\gamma$ interferon $\gamma$; PG, prostaglandin HPWEP, Helicobacter pylori water extract protein; FCS, fetal calf serum; $N F \kappa B$, nuclear factor $\kappa B ;$ MIP, macrophage inflammatory protein; ELISA, enzyme linked immunosorbent assay; RT-PCR, reverse transcriptionpolymerase chain reaction; TBS, Tris buffered saline; LPS, lipopolysaccharide; Th, T helper. 
Table 1 Primers and probes, and selected polymerase chain reaction (PCR) conditions

\begin{tabular}{|c|c|c|}
\hline PCR product & Temperature $\left({ }^{\circ} \mathrm{C}\right)^{*}$ & Primer sequence $\left(5^{\prime} \rightarrow 3^{\prime}\right)$ \\
\hline \multicolumn{3}{|l|}{ MIP- $1 \alpha$} \\
\hline Sense & 58 & 5'-TCA CCT GCT CAG AAT CAT GC-3' \\
\hline Antisense & & 5'-TCC ATA GAA GAG GTA GCT GTG G-3' \\
\hline \multicolumn{3}{|r|}{ - } \\
\hline Sense & 57 & 5'-AGC AGC TCA GTT CAG TTC CAG GTC A-3' \\
\hline Antisense & & 5'-CCA TGA AGC TCT GCG TGA CTG TCC T-3' \\
\hline \multicolumn{3}{|r|}{ - } \\
\hline Sense & 57 & 5'-ACC ATG AAC CCA AGT GCT GCC GTC-3' \\
\hline Antisense & & 5'-GCT TCA CTC CAG TTA AGG AGC CCT-3' \\
\hline \multicolumn{3}{|r|}{ (2) } \\
\hline Sense & 60 & 5'-ATG TTC CAT GTT TCT TIT AGG TAT ATC T-3' \\
\hline Antisense & & 5'-TGC ATT TCT CAA ATG CCC TAA TCC G-3' \\
\hline \multicolumn{3}{|r|}{ a } \\
\hline Sense & 57 & 5'-GGA CCC ATA TGT AAA AGA AGC AGA-3' \\
\hline Antisense & & 5'-TGT CAC TCT CCT CTT TCC AAT TCT-3' \\
\hline \multicolumn{3}{|r|}{ 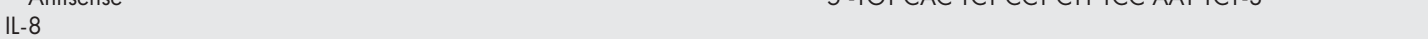 } \\
\hline Sense & 65 & 5'-AAG GAA CCA TCT CAC TG-3' \\
\hline Antisense & & 5'-GAT TCT TGG ATA CCA CAG AG-3' \\
\hline \multicolumn{3}{|l|}{ MCP-1 } \\
\hline Sense & 57 & 5'-TCC TGT GCC TGC TGC TCA TAG C-3' \\
\hline Antisense & & 5'-TTC TGA ACC CAC TTC TGC TTG G-3' \\
\hline \multicolumn{3}{|l|}{ RANTES } \\
\hline Sense & 65 & 5'-ATG AAG GTC TCC GCG GCA CGC-3' \\
\hline Antisense & & 5'-CTA GCT CAT CTC CAA AGA GTT-3' \\
\hline \multicolumn{3}{|l|}{ COX-2 } \\
\hline Forward & 60 & 5'-GCCAGAGTTTCACCGTAAATATGATT 3' \\
\hline Reverse & & 5'-GCGAGGGCCAGCTTTCA-3' \\
\hline Fluorogenic probe & & 5'-Fam-AAGTCCACCCCATGGCCCAGC-Tamra-3' \\
\hline \multicolumn{3}{|r|}{ 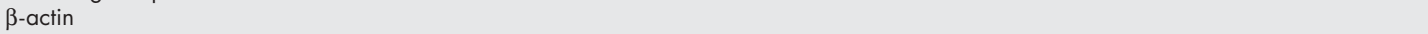 } \\
\hline Forward & 60 & 5'-TCACCCACACTGTGCCCATCTACGA 3' \\
\hline Reverse & & 5'-CAGCGGAACCGCTCATTGCCAATGG-3' \\
\hline Fluorogenic probe & & 5'-Fam-ATGCCC- Tamura-CCCCCATGCCATCCTGCGT-3' \\
\hline
\end{tabular}

\section{MATERIALS AND METHODS}

\section{Preparation of $\boldsymbol{H}$ pylori water extract protein}

A mixture of eight clinical isolates and strain NCTC 11637 were resuspended in distilled water, disrupted in a vortex agitator, and centrifuged. The supernatant was subjected to ion exchange chromatography by a stepwise method $(0,0.2,0.35$, and $0.5 \mathrm{~mol} / \mathrm{l}$ sodium phosphate buffer). The $0.35 \mathrm{~mol} / \mathrm{l}$ sodium phosphate fraction, containing a final protein concentration of $0.45 \mathrm{mg} / \mathrm{ml}$, was used as the $H$ pylori water extract protein (HPWEP).

\section{Preparation of media from MKN-28 gastric epithelial} cells in response to HPWEP

Confluent MKN-28 cells were incubated with RPMI 1640 medium supplemented with $10 \%$ fetal calf serum (FCS) at $37^{\circ} \mathrm{C}$ in the presence of HPWEP for 48 hours. Media separated by centrifugation for one minute at $10000 \mathrm{~g}$ were immediately added to $\mathrm{T}$ cells and cultured for 24 hours. In some experiments, media were stored at $-80^{\circ} \mathrm{C}$ until cytokine measurements.

\section{T cell culture and treatment}

Jurkat T cells were grown in complete RPMI 1640 medium supplemented with $10 \%$ FCS. Human peripheral T lymphocytes ( 11 H pylori uninfected healthy male volunteers, aged 31-45 years) were separated using paramagnetic beads (Dynabeads; Dynal, Oslo, Norway) coated with anti-CD3 antibody. Immunostaining with anti-CD3 antibody showed that 95\% of cells isolated by this method were peripheral T cells, consistent with results previously reported. ${ }^{18}$ Jurkat and peripheral $\mathrm{T}$ cells were both stimulated with each of the following: media obtained from MKN-28 cells, PMA (20 ng/ml; Sigma, St Louis, Missouri, USA), immobilised anti-CD3 antibody ( $0.3 \mu \mathrm{g} / \mathrm{ml}$; Neo Markers, Fremont, California, USA), recombinant human monocyte chemoattractant protein-1
(MCP-1), IL-7, IL-8, and RANTES (R\&D Systems, Minneapolis, Minnesota, USA) for 24 hours. A selective COX-2 inhibitor, $10 \mu$ M NS-398 (Taisho Pharmaceutical, Japan), or a selective COX-1 inhibitor, $0.03 \mu \mathrm{M}$ SC-560 (Pharmacia, New Jersey, USA), were added to peripheral T cell media one hour prior to stimulation. In some experiments, Jurkat $\mathrm{T}$ cells were also pretreated for one hour with the proteasome inhibitor MG-132 (Peptide Institute, Osaka, Japan) to prevent nuclear factor $\kappa \mathrm{B}(\mathrm{NF} \kappa \mathrm{B})$ activation.

\section{RT-PCR and cytokine measurements}

Total RNA was isolated from MKN-28 cells as per instructions in the Total RNA Isolation kit (Qiagen GmbH, Hilden, Germany). Reverse transcription-polymerase chain reactions (RT- PCR) were performed as previously described ${ }^{19}$ using the primers shown in table 1. Amplification products were visualised by ethidium bromide fluorescence in agarose gels. Concentrations of MCP-1, macrophage inflammatory protein (MIP)- $1 \alpha$, MIP- $1 \beta$, IL-7, IL-8, RANTES, and IFN- $\gamma$ in media from MKN-28 cells incubated with HPWEP were quantified using commercially available specific enzyme linked immunosorbent assay (ELISA) plates. Responses of peripheral $\mathrm{T}$ cell IL- 4 and IFN- $\gamma$ to the media or anti-CD3 antibody were also determined by ELISA. The plates were used according to instructions provided by the suppliers (MCP-1, MIP- $1 \alpha$, MIP$1 \beta$, IL-7, IL-8, and RANTES (RED Systems); IFN- $\gamma$ and IL-4 (Endogen, Cambridge, Massachusetts, USA)). MCP-1 levels were also examined in supernatants of gastric tissue sample homogenates $\left(10000 \mathrm{~g}\right.$ for 15 minutes at $4^{\circ} \mathrm{C}$ ) from $26 \mathrm{H}$ pylori gastritis and $12 \mathrm{H}$ pylori uninfected subjects. All subjects provided informed consent before endoscopy.

\section{Quantitative COX-2 mRNA analysis}

Real time quantitative $\mathrm{PCR}^{20}$ was performed to measure COX-2 mRNA expression levels in Jurkat $\mathrm{T}$ cells stimulated by media 
from HPWEP exposed MKN-28 cells. In brief, RNA isolated from Jurkat $\mathrm{T}$ cells as described above was reverse transcribed and subsequent cDNA amplified in the Model 7700 Sequence detector (PE Applied Biosynthesis, Perkin Elmer, Chiba, Japan) with primers, dual labelled fluorogenic probes, and a Taqman PCR Reagent Kit (Perkin Elmer, Branchburgh, New Jersey, USA). Primers and probes are described in table 1. Known concentrations of serially diluted COX-2 and $\beta$-actin cDNA generated by PCR were used as standards for quantification of sample cDNA. Copy numbers of cDNA for COX-2 were standardised to those for $\beta$-actin from the same sample.

\section{COX-1 and COX-2 protein expression and COX activity in $\mathrm{T}$ cells}

COX protein partially purified, as previously described, ${ }^{11}$ was visualised by western blotting using antihuman COX-1 antibody (diluted 1:25; IBL, Gunma, Japan) or COX-2 antibody (diluted 1:25; IBL). COX enzyme activity was determined using a crude T cell fraction, as described previously. ${ }^{1 .}$ Jurkat $\mathrm{T}$ cells incubated with agents for 24 hours were disrupted by sonication in ice cold $100 \mathrm{mM}$ Tris $\mathrm{HCl}(\mathrm{pH}$ 7.8) containing $1.0 \mathrm{mmol} / \mathrm{l}$ phenylmethylsulphonyl fluoride and $1.0 \mu \mathrm{mol} / \mathrm{l}$ pepstatin at $4^{\circ} \mathrm{C}$. Sonicates of $\mathrm{T}$ cells were centrifuged at $10000 \mathrm{~g}$ for five minutes and the resultant supernatant, containing both microsomal and cytosolic fractions, used as the enzyme source for measurement of COX activity. ${ }^{11}$ COX activity was expressed as the production of $\mathrm{PGE}_{2}$, as measured by ELISA (Assay Designs, Ann Arbor, Michigan, USA) in $\mathrm{pmol} / \mathrm{min} / \mathrm{mg}$ protein. The anti-MCP-1 neutralising antibody (R\&D Systems) completely suppressed human recombinant MCP-1 stimulated (150 pg/ml) COX activity in Jurkat $\mathrm{T}$ cells at 1:1000 titrations. Therefore, we used the neutralising antibody at that 1:1000 titration to test for MKN-28 media stimulated COX-2 mRNA expression and COX activity.

\section{Modified MTT assay}

Human peripheral $\mathrm{T}$ cell proliferation prepared from $1 \mathrm{l}$ $H$ pylori uninfected volunteers as described above was evaluated using a modified MTT assay, ${ }^{21}$ a tool known to be useful for quantifying viable cells. Specifically, the MTT assay is a colorimetric assay system which measures the reduction, by viable cell mitochondria, of tetrazolium components into insoluble formazan products. Briefly, $1 \times 10^{6} / \mathrm{ml} \mathrm{T}$ cells were cultured in RPMI on a 96 well plate in the presence of MKN-28 cell media, anti-CD3 antibody, or MCP-1 for 24 hours, and then each well incubated with $10 \mu \mathrm{l}$ MTT for 30 minutes. The reaction was stopped by addition of acidified Triton buffer. Samples were measured on a Bio-Rad plate reader at $595 \mathrm{~nm}$.

\section{Immunohistochemistry}

Three biopsy specimens obtained from the antrum and body were used for histological assessment. Serial $5 \mu \mathrm{m}$ sections were stained with haematoxylin-eosin and evaluated using the updated Sydney system. Three slides were prepared, consisting of one antrum and two body tissue specimens, for each patient. When the grade was different between the three slides, the median value was obtained as the representative grade. This median value for each patient was used for calculation of mean values.

For COX-2 immunostaining in the gastric mucosa, $3 \mu \mathrm{m}$ sections were deparaffinised and endogenous peroxidase activity blocked with $5 \% \mathrm{H}_{2} \mathrm{O}_{2}$ in Tris buffered saline (TBS). Non- specific binding was blocked with $5 \%$ rabbit serum in TBS and tissues incubated with anti- COX-2 antibody (IBL; dilution 1:100) in TBS containing 1\% bovine serum albumin for two hours. We quantified COX-2 expression levels in gastric tissue samples by counting mononuclear cells expressing COX-2 and evaluating the staining intensity for $26 \mathrm{H}$ pylori gastritis and $12 \mathrm{H}$ pylori uninfected subjects. Overall intensity was arbitrarily graded as 0 (negative), 1 ( $<5 \%$ cells with positive staining), $2(5-30 \%), 3(30-60 \%$, with strong staining), and $4\left(>60 \%\right.$, with very strong staining). ${ }^{22}$ Then, the relationship between COX-2 intensity and MCP-1 levels was examined in $26 \mathrm{H}$ pylori gastritis subjects. For MCP-1 immunostaining in the gastric mucosa, tissue samples were immediately embedded in OCT compound. Serial sections were incubated overnight with polyclonal rabbit anti-human MCP- 1 antibody (1:100). After washing, bound antibody was detected using the LSAB 2 kit (Dako, Carpinteria, California, USA) with diaminobenzidine as the chromogen. As negative controls, primary antibodies were replaced with isotype matched immunoglobulin.

Double labelling immunofluorescence methods and confocal laser scanning microscopy were used to evaluate the colocalisation of immunoreactivity for the pair of mouse antihuman COX-2 (IBL; dilution 1:20) and rabbit antihuman CD3 (Dako; dilution 1:20). Sections were incubated overnight at $4^{\circ} \mathrm{C}$ with a mixture of the two primary antibodies, and then with FITC or Texas red conjugated secondary antibodies (horse antimouse IgG (Vector Laboratories, Burlingame, California, USA) dilution 1:100 and goat antirabbit IgG (Vector) dilution 1:100, for COX-2 and CD3, respectively) followed by nuclear counterstaining with 4', 6-diamidino- 2-phenylindole (DAPI; Sigma Chemical) for 15 minutes.

\section{Statistical analysis}

Results are expressed as mean (SD). For statistical evaluation of group data, a Students' $t$ - test for paired data and analysis of variance (ANOVA) for multiple comparisons were followed by Scheffe's F test. A p value of less than 0.05 was statistically significant.

\section{RESULTS}

COX protein expression in peripheral $\mathrm{T}$ cells and Jurkat $\mathrm{T}$ cells stimulated by $\mathrm{MKN}-28$ cell media

COX-2 expression (lanes c and $f$ in fig 1) was clearly induced in both $\mathrm{T}$ cell types stimulated by media from HPWEP exposed MKN-28 cells (fig 1A). In contrast, COX-2 expression was evident only as a faint band in both $\mathrm{T}$ cell types when they were directly stimulated with HPWEP (lanes d and g). No COX-2 expression was detected in unstimulated $\mathrm{T}$ cells (lanes e and h). To exclude the possibility that COX-2 expression in peripheral $\mathrm{T}$ cells is mainly due to macrophage contamination during peripheral T cell preparations, we stimulated peripheral $\mathrm{T}$ cells with lipopolysaccharide (LPS) $(1 \mu \mathrm{g} / \mathrm{ml})$. However, we detected no LPS stimulated COX-2 expression in peripheral T cells (data not shown). COX-1 expression levels did not vary for stimulated and unstimulated T cells (fig lB).

\section{COX activity in stimulated Jurkat $\mathrm{T}$ cells}

Media from MKN-28 cells incubated with HPWEP induced a significant increase in COX activity in Jurkat T cells (fig 2). Jurkat $\mathrm{T}$ cells directly stimulated with HPWEP also showed a small increase in COX activity. These results suggest that in response to HPWEP, MKN-28 cells secrete chemokines involved in the increase in COX-2 protein expression and COX activity in Jurkat $\mathrm{T}$ cells.

\section{Stimulated cytokine mRNA expression in MKN-28 cells} and protein release into the media

Next we measured mRNA levels for several cytokines by specific RT-PCR (fig 3A). MCP-1, IL-7, and IL-8 mRNA expression was stimulated by HPWEP while RANTES mRNA levels were not significantly changed by HPWEP stimulation. Furthermore, IFN- $\gamma$, MIP-1 $\alpha$, MIP-1 $\beta$, and IP-10 mRNA were not expressed in MKN-28 cells incubated with or without HPWEP. We also measured MCP-1, IL-7, and IL-8 media levels by specific ELISA (fig 3B). The stimulated media contained significant levels of MCP-1 (133 (6.9) pg/ml), IL-7 (2.8 (1.2) 
A

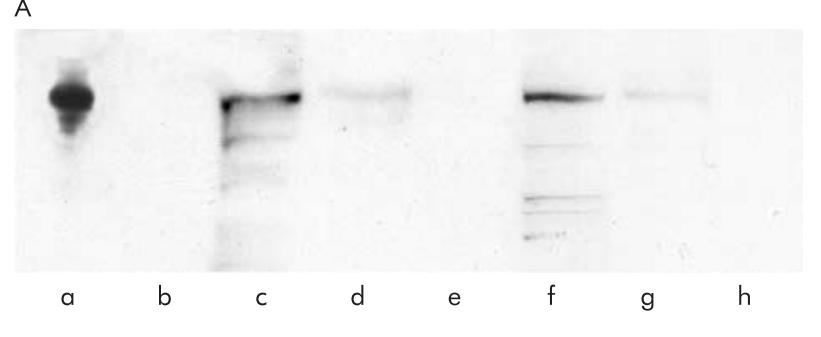

B

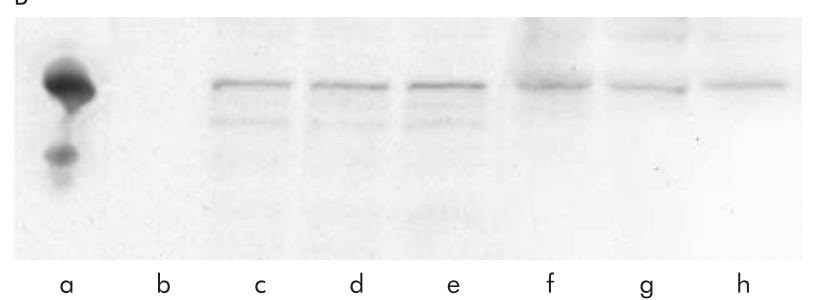

Figure 1 Cyclooxygenase (COX) protein expression in peripheral $\mathrm{T}$ cells and Jurkat $\mathrm{T}$ cells stimulated by MKN-28 cell media. (A) COX-2 protein expression in T cells stimulated by MKN-28 cell media. COX-2 expression in peripheral T cells incubated with media from Helicobacter pylori water extract protein (HPWEP) exposed MKN-28 cells (lane c), HPWEP (lane d), and unstimulated MKN-28 cell media (lane e). Lanes $\mathrm{f}-\mathrm{h}$ : as in lanes a-e except that Jurkat $\mathrm{T}$ cells instead of peripheral $T$ cells are represented. Lanes $a$ and $b$ indicate COX-2 positive control $(70 \mathrm{kDa})$ and COX-1 positive control $(68 \mathrm{kDa})$, respectively. (B) COX-1 protein expression in $\mathrm{T}$ cells stimulated by media from MKN-28 cells. Lane a, COX-1 positive control; lane b, COX-2 positive control; lanes $c-h$, as in (A) except that COX-1 instead of COX-2 expression is represented. Each panel is representative of four separate experiments.

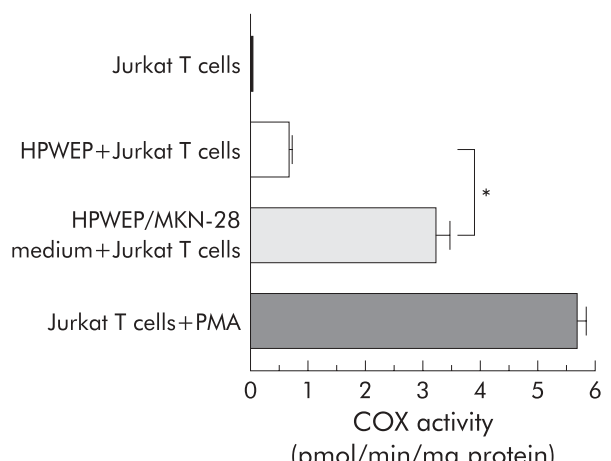

(pmol/min/mg protein)

Figure 2 Comparison of cyclooxygenase (COX) activity in Jurkat T cells. COX activity in stimulated and unstimulated Jurkat $T$ cells was determined as described in materials and methods. Jurkat T cells were stimulated with Helicobacter pylori water extract protein (HPWEP+Jurkat T cells), media from HPWEP exposed MKN-28 cells (HPWEP/MKN-28 medium+Jurkat T cells), and $20 \mathrm{ng} / \mathrm{ml}$ PMA (Jurkat $T$ cells $+P M A)$, respectively. Prostaglandin $E_{2}$ generated in each sample was determined in duplicate. Each value represents the mean (SEM) of four separate experiments. ${ }^{*} p<0.05$.

$\mathrm{pg} / \mathrm{ml})$, and IL-8 (40.5 (14.5) pg/ml). However, ELISA tests for other cytokines in the media disclosed only one other cytokine, RANTES, and this at the same level as in unstimulated media (data not shown). The presence of MCP-1 in media from HPWEP exposed MKN-28 cells was also confirmed by western blot analysis (data not shown).

\section{MCP-1 stimulated and MG-132 abrogated COX-2} expression in Jurkat $T$ cells

MCP-1 stimulated COX-2 expression in Jurkat T cells at 100 $\mathrm{pg} / \mathrm{ml}$, a concentration consistent with that of the media (fig 4). However, the level of COX-2 expression stimulated by MCP-1 seemed lower than expected, suggesting that other
A
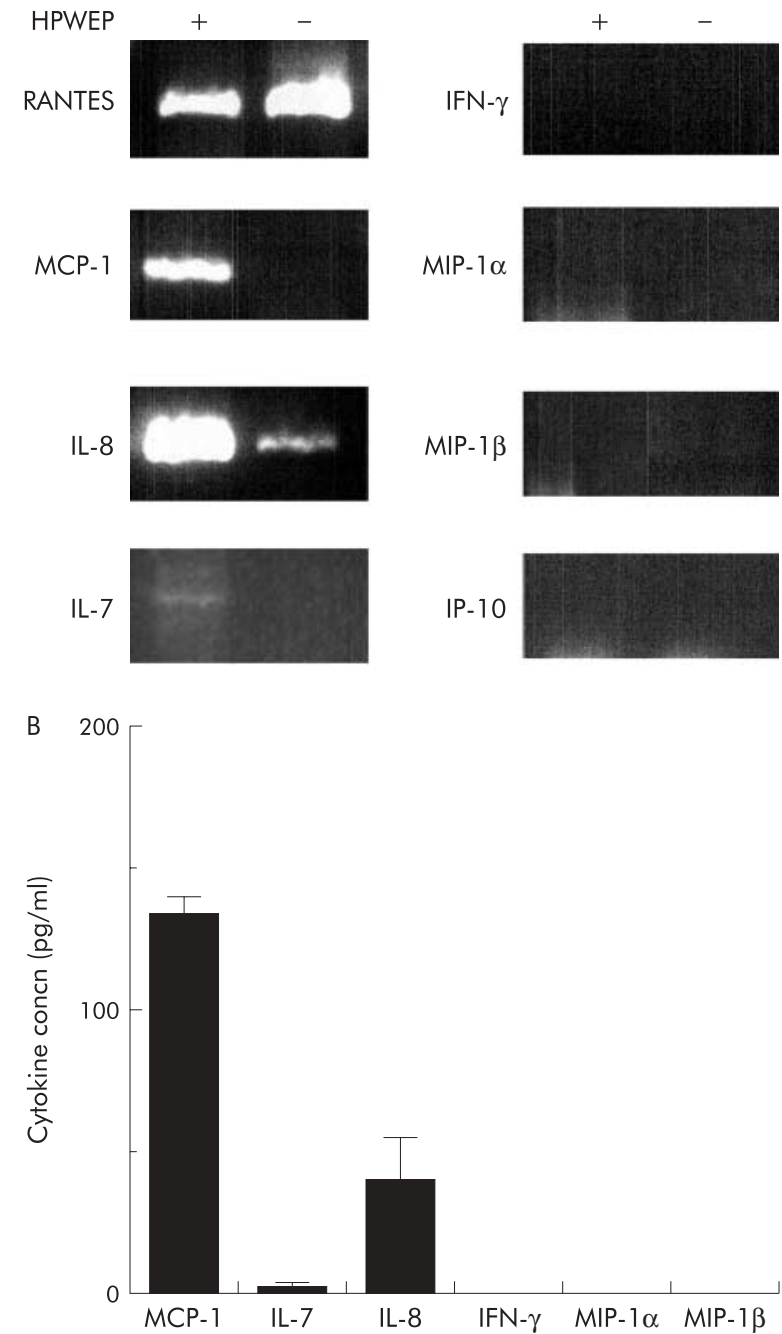

Figure 3 mRNA and protein expression of cytokines in MKN-28 cells and their release into the media. (A) Monocyte chemoattractant protein 1 (MCP-1), interleukin (IL)-7, RANTES, and IL-8 mRNAs were found in Helicobacter pylori water extract protein (HPWEP) exposed MKN-28 cells. In contrast, interferon $\gamma($ IFN- $\gamma$ ), macrophage inflammatory protein (MIP)- $1 \alpha$, MIP-1 $\beta$, and IP- 10 mRNAs were not expressed. Each panel is representative of four separate experiments. (B) Cytokine levels were determined as described in materials and methods. Each cytokine was determined in duplicate for each sample. Values are mean (SEM) of four separate experiments.

factors are also involved in COX-2 expression in Jurkat T cells. Pretreatment of Jurkat T cells with MG-132 abrogated both MKN-28 cell media and MCP-1 stimulated COX-2 expression in Jurkat T cells. On the other hand, IL-7 at $5 \mathrm{pg} / \mathrm{ml}$, IL-8 at 40 $\mathrm{pg} / \mathrm{ml}$, and RANTES at $600 \mathrm{pg} / \mathrm{ml}$, concentrations identified in MKN-28 media, did not stimulate COX-2 expression in Jurkat $\mathrm{T}$ cells, as determined by western blot analysis.

COX-2 mRNA levels and COX activity in Jurkat T cells were inhibited by anti-MCP-1 neutralising antibody

Media from MKN-28 cells incubated with HPWEP induced a significant increase in COX- $2 / \beta$-actin mRNA levels in Jurkat T cells (fig 5). Jurkat $\mathrm{T}$ cells directly stimulated with HPWEP also showed a moderate increase in COX-2/ $\beta$-actin mRNA. Anti-MCP-1 neutralising antibody (1:1000 titration) significantly suppressed COX-2 mRNA levels in Jurkat T cells stimulated with the MKN-28 media, suggesting that MCP-1 in the media stimulated COX-2 mRNA expression in Jurkat T cells. Then we investigated whether the neutralising antibody could 


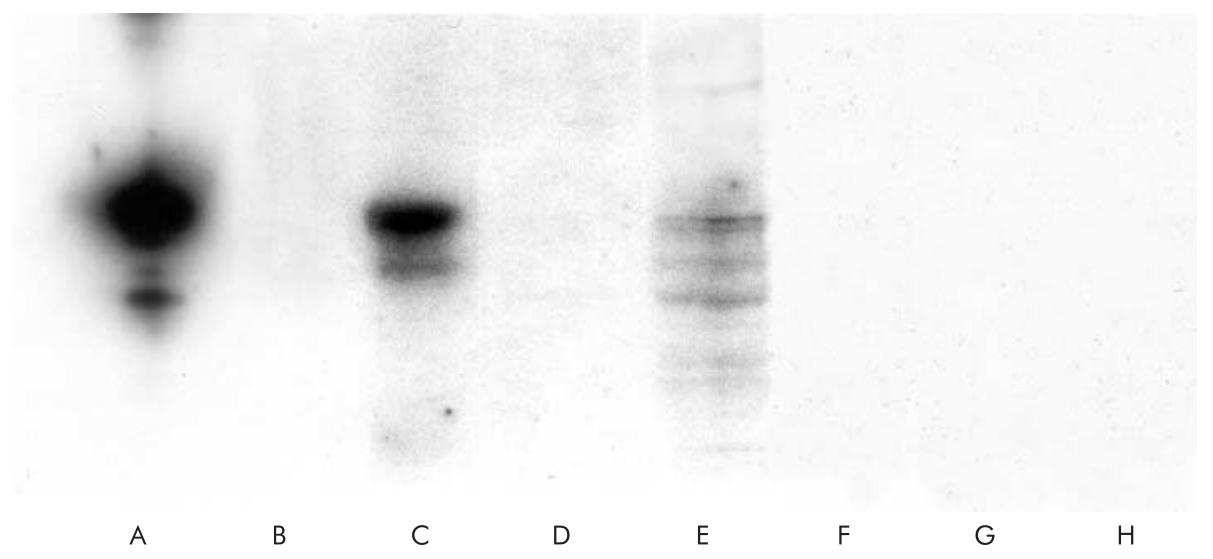

Figure 4 Effects of cytokines and MG-132 on Jurkat T cell cyclooxygenase 2 (COX-2) protein expression. Lane A, COX-2 positive control; lane B, COX-1 positive control; lane C, COX-2 protein expression in Jurkat T cells incubated with media from Helicobacter pylori water extract protein (HPWEP) exposed MKN-28 cells; lane D, COX-2 protein expression in Jurkat T cells pretreated with MG-132 and then incubated with media from HPWEP stimulated MKN-28 cells; lane E, COX-2 protein expression in Jurkat T cells stimulated with monocyte chemoattractant protein 1 at $100 \mathrm{pg} / \mathrm{ml}$; lanes $\mathrm{F}-\mathrm{H}$, lack of COX-2 protein expression in Jurkat T cells stimulated with human recombinant IL-8 $40 \mathrm{pg} / \mathrm{ml}$ (lane F), IL-7 $5 \mathrm{pg} / \mathrm{ml}$ (lane G), and RANTES $600 \mathrm{pg} / \mathrm{ml}$ (lane H). Experiments were repeated four times and the panel shown is a representative experiment.

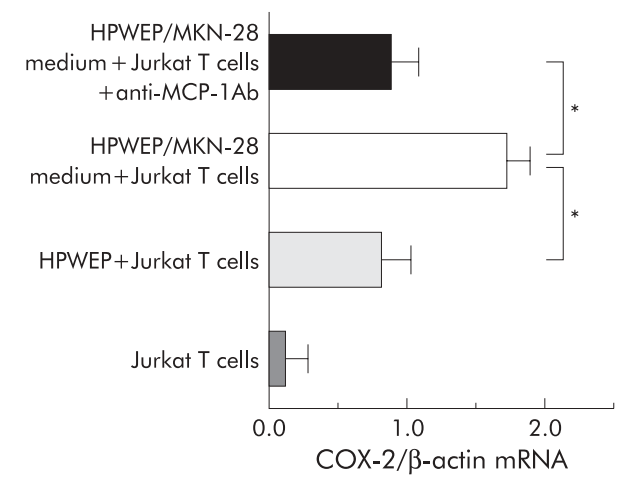

Figure 5 Comparison of cyclooxygenase 2 (COX-2) mRNA production in Jurkat T cells. COX-2/ $\beta$-actin mRNA levels for stimulated Jurkat $\mathrm{T}$ cells were determined by real time polymerase chain reaction, as described in materials and methods. COX-2/ $\beta$-actin mRNA levels of Jurkat T cells increased following incubation with Helicobacter pylori water extract protein (HPWEP) or HPWEP/MKN-28 medium (as in fig 2) and were suppressed by preincubation with anti-monocyte chemoattractant protein 1 (MCP-1) neutralising antibody (HPWEP/MKN-28 medium+Jurkat T cells+anti-MCP-1Ab). Each value represents the mean (SEM) of four separate experiments. ${ }^{\star} p<0.05$.

actually suppress COX activity in Jurkat T cells. Anti-MCP-1 neutralising antibody (1:1000 titration) significantly suppressed COX activity by $38 \%$ in Jurkat T cells stimulated with MKN-28 cell media but a higher concentration of anti-MCP-1 neutralising antibody (1:300 titration) showed no further suppression of COX activity (39\%). In contrast, normal rabbit serum IgG ( $1 \mathrm{mg} / \mathrm{ml})$ had no effect on COX activity in media stimulated Jurkat T cells.

\section{Proliferation of peripheral T cells}

Proliferation of peripheral $\mathrm{T}$ cells treated with stimulated MKN-28 cell media and anti-CD3 antibody as a positive control significantly increased (188 (6)\% and 308 (16)\%, respectively) compared with those treated with unstimulated MKN-28 media alone. NS-398 significantly inhibited proliferation of peripheral $\mathrm{T}$ cells (157 (6)\%) stimulated by the media, while SC-560 did not suppress proliferation of these T cells. Recombinant MCP-1 protein significantly increased proliferation of peripheral T cells (161 (6)\%). NS-398 also significantly inhibited proliferation of peripheral $\mathrm{T}$ cells stimulated with MCP-1 while SC-560 had no effect, suggesting that
MCP- 1 released from MKN-28 cells is involved in T cell proliferation via COX-2 activation (fig 6A).

\section{IFN- $\gamma$ and IL-4 in supernatant from cultured peripheral T cells}

IFN- $\gamma$ concentrations in supernatants from peripheral $\mathrm{T}$ cells significantly increased in response to stimulation by media from HPWEP exposed MKN-28 cells (172 (15.8) pg/mg protein) while IL-4 concentrations (2.0 (0.8) pg/mg protein) did not significantly increase in response to stimulation. IFN- $\gamma$ concentrations also increased in response to stimulation by anti-CD3 antibody (218.3 (24.8) pg/mg protein) whereas IL-4 failed to respond (1.8 (0.9) pg/mg protein). NS-398 significantly inhibited this media stimulated IFN- $\gamma$ release (134.2 (11.2) $\mathrm{pg} / \mathrm{mg}$ protein) whereas SC-560 did not significantly inhibit IFN- $\gamma$ release $(161.8$ (14.1) pg/mg protein). There was no increase in basal IFN- $\gamma$ levels $(27.2(6.2) \mathrm{pg} / \mathrm{mg}$ protein) in response to T cell stimulation by MCP-1 at $100 \mathrm{pg} / \mathrm{ml}$ (fig 6B) whereas IL-4 concentration (22.5 (1.9) pg/mg protein) significantly increased. NS-398 significantly inhibited this MCP-1 stimulated IL-4 release (14.6 (1.3) pg/mg protein) while SC-560 had no effect on IL-4 release (20.2 (2.5) pg/mg protein) (fig 6C).

\section{Distribution of COX-2 positive T cells and MCP-1 positive cells in the gastric mucosa}

FITC labelled (green) cells in the lamina propria in fig 7A show COX-2 immunoreactivity. Figure 7B shows mucosal T cells labelled with Texas red conjugated anti-CD3 antibodies for the same section. Double immunostaining for COX-2 and mucosal T cells demonstrated the presence of COX-2 positive mucosal $\mathrm{T}$ cells in the lamina propria of $H$ pylori infected gastritis mucosa (fig 7C). In contrast, there were no COX-2 positive $\mathrm{T}$ cells in $H$ pylori uninfected gastritis mucosa, and just a few CD3 positive cells (fig 7D). In fig 7E, we can see MCP-1 immunoreactivity in surface epithelial cells, as well as in a number of mononuclear cells.

\section{Correlation between MCP-1 levels and intensity of COX-2 expressions in gastric mucosal samples}

MCP-1 levels were significantly greater in H pylori infected tissue samples (166.1 (32.6) $\mathrm{pg} / \mathrm{mg}$ protein) than in uninfected mucosal samples (81.6 (7.7) pg/mg protein). There was a significant correlation $(r=0.869, \mathrm{p}<0.0001)$ between intensity of mononuclear cell infiltration and MCP-1 levels in gastric mucosal samples from patients with and without $H$ pylori 


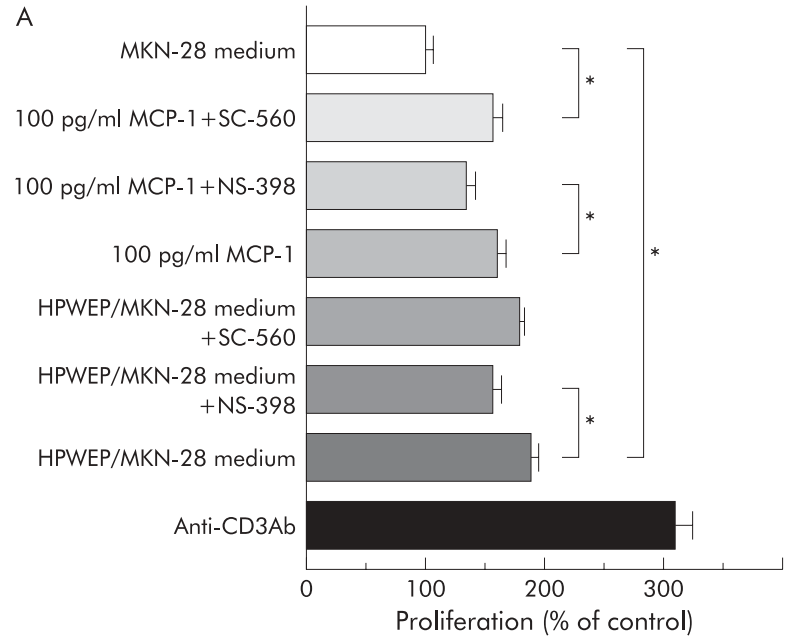

B

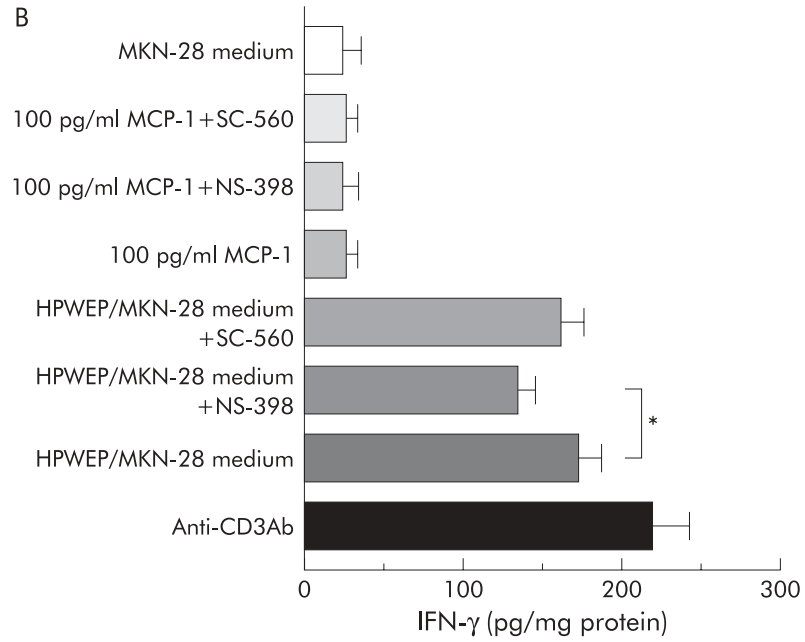

C

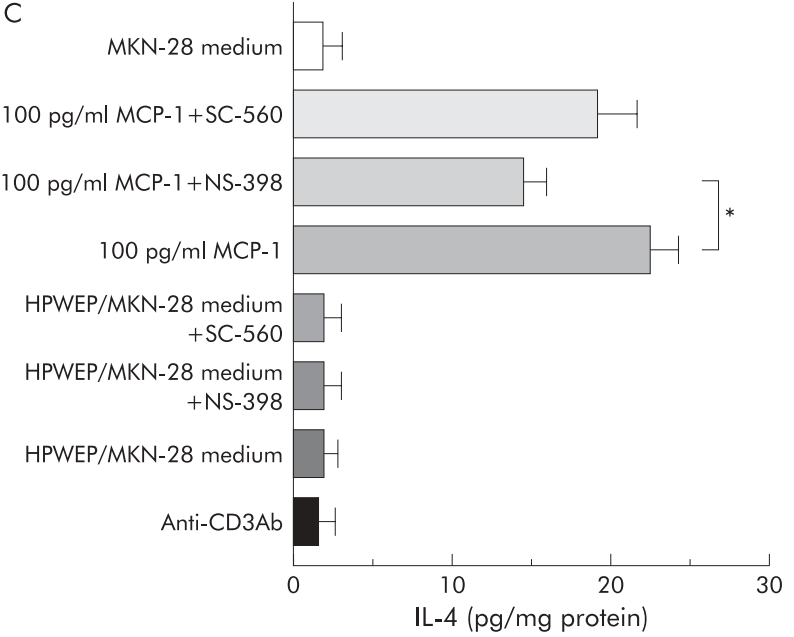

Figure 6 T cell cytokine production and proliferation.

(A) Proliferation of activated peripheral T cells was determined as described in materials and methods. Peripheral T cells were stimulated by anti-CD3 antibody $(0.3 \mathrm{\mu g} / \mathrm{ml})$, Helicobacter pylori water extract protein (HPWEP)/MKN-28 medium, HPWEP/MKN-28 medium+NS-398 (HPWEP/MKN-28 medium in the presence of 10

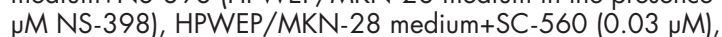
$100 \mathrm{pg} / \mathrm{ml}$ monocyte chemoattractant protein 1 (MCP-1), 100 $\mathrm{pg} / \mathrm{ml} \mathrm{MCP}-1+\mathrm{NS}-398(10 \mu \mathrm{M})$, and $100 \mathrm{pg} / \mathrm{ml} \mathrm{MCP}-1+$ SC-560 (0.03 $\mu \mathrm{M})$. MKN-28 medium, peripheral T cells treated with unstimulated MKN-28 media. For each MTT assay, samples were determined in triplicate. ${ }^{*} p<0.05$. (B) Interferon $\gamma$ (IFN- $\gamma$ ) production was determined as described in materials and methods. (C) Interleukin 4 (IL-4) production was determined as described in material and methods. Each value represents the mean (SEM) of 11 separate experiments. infection. We also found that in vitro, MCP-1 induced COX-2 expression in T cells which led us to investigate whether there was a significant relationship between MCP-1 levels and intensity of COX-2 expression. We found a significant correlation $(r=0.835, \mathrm{p}<0.0001)$ between intensity of COX-2 expression and MCP-1 levels in gastric mucosal samples when COX-2 expression levels were quantitatively evaluated (fig 8).

\section{DISCUSSION}

In this study, we investigated the role of $H$ pylori induced cytokine release from gastric epithelial cells in T cell COX-2 expression and activation, in vitro and in vivo. Several lines of evidence in the present study suggest that MKN-28 cells, in response to HPWEP stimulation, secreted various cytokines, including MCP-1, and thus induced T cell COX-2 expression and activity. Firstly, media from HPWEP exposed MKN-28 cells stimulated COX-2 mRNA and protein expression in T cells. Secondly, RT-PCR and specific ELISA showed that in MKN-28 cells, MCP-1 mRNA was expressed and MCP-1 protein released in response to HPWEP. Thirdly, in Jurkat T cells, MCP-1 stimulated COX-2 expression levels and COX activity while anti-MCP-1 neutralising antibody suppressed both COX-2 mRNA expression and COX activity stimulated by MKN-28 cell media. Therefore, MCP- 1 seems to play a role in COX-2 expression in T cells. Although other studies to date have shown that IL- $8,{ }^{2}$ a CXC chemokine released from gastric epithelial cells, may be involved in mucosal neutrophil infiltration, few have considered the role of MCP-1 expression, a C-C chemokine, in gastric epithelial cells. ${ }^{23}$ As far as we know, this is the first report to show a relationship between MCP-1 release from gastric epithelial cells and induction of COX-2 expression leading to T cell activation. However, COX activity in media stimulated Jurkat $\mathrm{T}$ cells was not completely suppressed by anti-MCP-1 neutralising antibody. This suggests that other cytokines in the MKN-28 cell media are also involved in COX-2 protein expression and COX activity in T cells. Recently, CXC chemokines as well as C-C chemokines have been shown to act as chemoattractants for $\mathrm{T}$ cells and to induce cytokine production from $\mathrm{T}$ cells. ${ }^{24}$ However, in the present study, we were not able to detect any MIP-l $\alpha$ or MIP- $1 \beta$ in the stimulated media. Furthermore, IL-7, IL-8, or RANTES did not stimulate Jurkat $\mathrm{T}$ cell COX- 2 protein expression. Thus it appears that in addition to MCP-1, other factors may be involved in T cell COX-2 expression. As media MCP-1 could not stimulate COX-2 protein expression in Jurkat T cells pretreated with MG-132, it appears that MCP-1 may stimulate COX-2 expression via NFKB activation.

We also found that COX-2 induction, as seen with media from MKN-28 cells and MCP-1, might play an important role in peripheral T cell cytokine production and proliferation. NS398 , a specific COX-2 inhibitor, induced a moderate reduction in $\mathrm{T}$ cell proliferation, whether stimulated by media or MCP- 1 alone.

In parallel with media induced peripheral $\mathrm{T}$ cell proliferation, IFN- $\gamma$ was also released from peripheral $\mathrm{T}$ cells in response to the media. This IFN- $\gamma$ release was again partially inhibited by NS-398, suggesting that COX-2 is also involved in producing IFN- $\gamma$, a major cytokine linked to functional T cell polarisation toward a $\mathrm{T}$ helper $\mathrm{l}$ (Thl) profile. Thus media from HPWEP exposed MKN-28 cells appear to shift T cells in a Thl direction. These data are consistent with a recent report suggesting that $H$ pylori induced mucosal inflammation is mediated by Thl predominance. ${ }^{25}$ On the other hand, MCP-1, which also stimulated COX-2 expression and COX-2 dependent $\mathrm{T}$ cell proliferation, was found in the present study to stimulate IL-4 secretion from peripheral $\mathrm{T}$ cells while having no effect on IFN- $\gamma$ secretion. This suggests that MCP- 1 alone is linked to Th2 polarisation. ${ }^{26}{ }^{27}$ The results of the present study are also consistent with previous studies linking MCP-1 to Th2 polarisation. Although we do not know why media containing 

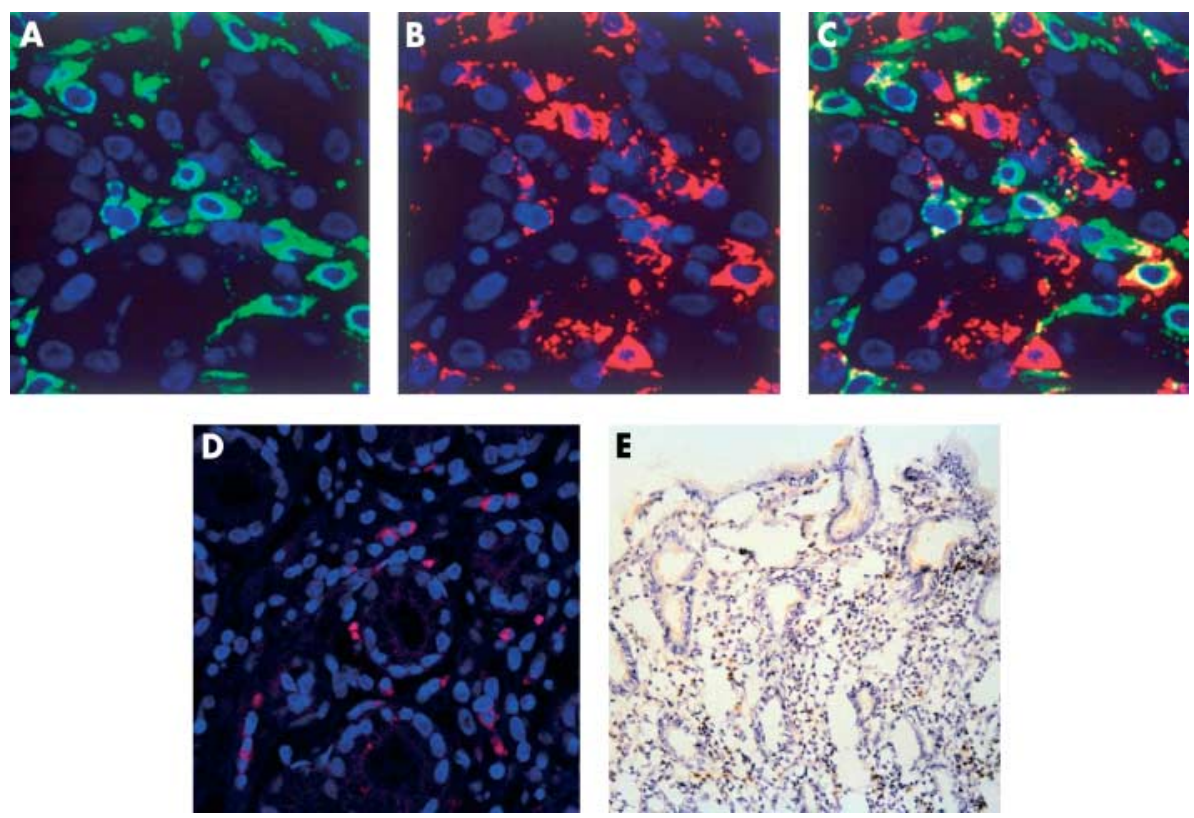

Figure 7 Localisation of cyclooxygenase 2 (COX-2) positive mucosal T cells and monocyte chemoattractant protein 1 (MCP-1) positive cells in the gastric mucosa. (A) COX-2 positive cells in the lamina propria of Helicobacter pylori infected gastritis (original magnification, $\times 150$ ). (B) CD3 positive cells in the lamina propria of $H$ pylori infected gastritis (original magnification, $\times 150$ ). (C) Double positive staining (yellow cells) reveals COX-2 positive mucosal T cells in $H$ pylori infected gastritis (original magnification, $\times 150$ ). (D) Double staining of $H$ pylori uninfected gastritis mucosa with anti-COX-2 antibody and anti-CD3 antibody (original magnification, $\times 90$ ). (E) MCP-1 positive epithelial cells and mononuclear cells in the lamina propria, as seen in $\mathrm{H}$ pylori infected gastritis (original magnification, $\times 200$ ).

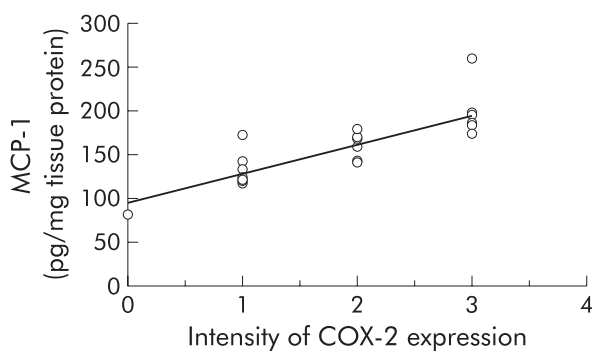

Figure 8 Correlation between monocyte chemoattractant protein 1 (MCP-1) levels and intensity of cyclooxygenase 2 (COX-2)

expression in gastric mucosa. Pearson's correlation analysis was used to assess the correlation between MCP-1 levels and intensity of COX-2 expression in Helicobacter pylori infected gastritis.

MCP-1 failed to stimulate IL-4 secretion from peripheral T cells, other factors in the media might be involved in IFN- $\gamma$ secretion and IL-4 inhibition in these cells. Alternatively, it is possible that IFN- $\gamma$ downregulates CD30, a marker of IL-4 response. ${ }^{28}$ In the present study, we also found that COX-2 expression in $\mathrm{T}$ cells was apparently linked to both Thl and Th2 polarisation. NS-398 partially inhibited IFN- $\gamma$ release stimulated by media from HPWEP exposed MKN-28 cells. In addition, NS-398 also induced a moderate reduction in MCP-1 and media stimulated peripheral $\mathrm{T}$ cell proliferation and IL-4 release. Thus although COX-2 expression in peripheral T cells induced by cytokines released from gastric epithelial cells plays an important role in $\mathrm{T}$ cell function, it seems to have no significant effect on $\mathrm{T}$ cell polarisation. The role of COX-2 in T cell activation has recently been shown in humans. ${ }^{29}$ These recent studies suggested that COX-2 in T cells may be linked to both Th1 and Th2 polarisation. ${ }^{29} 30$

Gilroy et al have also reported that in their model, COX-2 may regulate resolution of acute inflammation by generating an alternative set of anti-inflammatory prostaglandins. ${ }^{31}$ In a new study on coeliac disease by Kainulainen et al, COX-2 positive $\mathrm{T}$ cells were found in the lamina propria of mucosal lesions. ${ }^{17}$ In our current study, in addition to COX-2 expression in peripheral $\mathrm{T}$ cells in vitro, we also found $\mathrm{COX}-2$ positive $\mathrm{T}$ cells infiltrating into the gastric mucosa in vivo. All things considered, we suggest that in chronic $H$ pylori infected gastric mucosa, COX-2 might be involved in the immunomodulatory response, although we have yet to establish its exact role.

Previous studies have reported that MCP-1 is localised in epithelial cells of the colon and that its expression correlates with $\mathrm{T}$ cell infiltration in inflammatory bowel disease mucosa. ${ }^{32}{ }^{33}$ A previous study using PCR analysis indicated possible MCP-1 expression in a gastric epithelial cell line. ${ }^{23} \mathrm{We}$ demonstrated in the present study that MCP-1 is in fact released from gastric epithelial cell lines in response to HPWEP. In addition, we found for the first time that MCP-1 was localised mainly in gastric epithelial cells and also partly in mesenchymal cells of $H$ pylori infected mucosa. MCP-1 immunoreactivity was limited to surface epithelial cells, with no MCP-1 immunoreactivity seen in either glandular cells or $H$ pylori uninfected epithelial cells. This suggests that $H$ pylori in proximity to pit cell surfaces might affect MCP-1 expression in the gastritis mucosa.

MCP-1 levels in these gastritis tissue samples were closely related to intensity of COX-2 expression, consistent with our in vitro findings that MCP-1 stimulated increases in COX-2 expression levels in T cells. This leads us to hypothesise that MCP-1 released from gastric epithelial cells triggers COX-2 induction and $\mathrm{T}$ cell infiltration in $H$ pylori infected gastric mucosa. However, it is not yet known whether MCP-1 released from gastric epithelial cells is actually involved in Th2 polarisation in gastritis mucosa in vivo.

\section{ACKNOWLEDGEMENTS}

This work was supported in part by grants from the Ministry of Education, Culture, and Science and from the Ministry of Health, Japan.

\section{Authors' affiliations}

S Futagami, T Hiratsuka, A Tatsuguchi, K Suzuki, M Kusunoki, Y Shinii, K Shinoki, T lizumi, T Akamatsu, H Nishigaki, K Wada, K Miyake, K Gudis, T Tsukui, C Sakamoto, Third Department of Internal Medicine, Nippon Medical School, Tokyo, Japan 


\section{REFERENCES}

1 Keates S, Sougioultzis S, Keates AC, et al. Cag + Helicobacter pylori induce transactivation of the epidermal growth factor receptor in AGS gastric epithelial cells. J Biol Chem 2001;276:48127-34.

2 Crowe SE, Alvarez L, Dytoc M, et al. Expression of interleukin 8 and $C D$ 54 by human gastric epithelium after Helicobacter pylori infection in vitro. Gastroenterology 1995; 108:65-74.

3 Shimoyama T, Everett SM, Dixon MF, et al. Chemokine mRNA expression in gastric mucosa is associated with Helicobacter pylori cagA positivity and severity of gastritis. J Clin Pathol 1998;51:765-70.

4 D'Elios MM Manghetti M Carli MD, et al. T helper 1 effector cells specific for Helicobacter pylori in the gastric antrum of patients with peptic ulcer disease. J Immunol 1997;158:962-7.

5 Abe N, Katamura K, Shintaku N, et al. Prostaglandin $E_{2}$ and IL-4 provide native CD4+ T cells with distinct inhibitory signals for the priming of IFN- $\gamma$ production. Cell Immunol 1997; 181:86-92.

6 Newberry RD, Stenson WF, Lorenz RG. Cyclooxygenase-2-dependent arachidonic acid metabolites are essential modulators of the intestinal immune response to dietary antigen. Nat Med 1999;5:900-6.

7 Hwang D, Jang BC, Yu G, et al. Expression of mitogen-induciable cyclooxygenase induced by lipopolysaccharide: mediation through both mitogen-activated protein kinase and NF-kappa B signaling pathways in macrophages. Biochem Pharmacol 1997:54:87-96.

8 Dubois RN, Abramson SB, Crofford L, et al. Cyclooxygenase in biology and disease. FASEB J 1998;12:1063-73.

9 Tsujii $M$, Kawano S, DuBois RN. Cyclooxygenase-2 expression in human colon cancer cells increases metastatic potential. Proc Natl Acad Sci U S A 1997; 94:3336-40.

10 Tsujii M, Kawano S, Tsuji S, et al. Cyclooxygenase regulates angiogenesis induced by colon cancer cells. Cell 1998:93:705-16.

11 Mizuno H, Sakamoto C, Matsuda K, et al. Induction of cyclooxygenase 2 in gastric mucosal lesions and its inhibition by the specific antagonist delays healing in mice. Gastroenterology 1997;112:387-97.

12 Futagami S, Hiratsuka T, Wada K, et al. Inhibition of Helicobacter pylori-induced cyclooxygenase-2 aggravates NSAID-caused gastric damage in Mongolian gerbils. Aliment Pharmacol Ther 2002:16:847-55.

13 Tatsuguchi A, Sakamoto C, Wada K, et al. Localisation of cyclooxygenase 1 and cyclooxygenase 2 in Helicobacter pylori related gastritis and gastric ulcer tissues in humans. Gut 2000;46:782-9.

14 Iniguez MA, Punzon C, Fresno M. Induction of cyclooxygenase-2 on activated T lymphocytes: Regulation of T cell activation by cyclooxygenase-2 inhibitors. J Immunol 1999:163:111-19.

15 Eaton KA, Mefford M, Thevenot T. The role of T cell subsets and cytokines in the pathogenesis of Helicobacter pylori gastritis in mice. $J$ Immunol 2001; 166:7456-61.

16 Mohammadi $M$, Nedrud J, Redline R, et al. Murine CD4 T-cell response to Helicobacter infection: Th 1 cells enhance gastritis and Th2 cells reduce bacterial load. Gastroenterology 1997;113:1848-57.
17 Kainulainen $\mathbf{H}$, Rantala I, Collin P, et al. Blisters in the small intestinal mucosa of celiac patients contain T cells positive for cyclooxygenase 2 . mucosa of celiac patie

18 Quiding-Jarbrink M, Ahlstedt I, Lindholm C, et al. Homing commitment of lymphocytes activated in the human gastric and intestinal mucosa. Gut 2001;49:519-25.

19 Shimoyama T, Everett SM, Fukuda S, et al. Influence of smoking and alcohol on gastric chemokine mRNA expression in patients with Helicobacter pylori infection. J Clin Pathol 2001;54:332-4.

20 Heid CA Stevens J, Livak KJ, et al. Real time quantitative PCR. Genome Res 1996;6:986-94.

21 Tominaga $\mathbf{H}$, Ishiyama $M$, Ohseto $F$, et al. A water-soluble tetrazolium salt useful for colorimetric cell viability assay. Anal Commun 1999;36:47-50.

22 Sung JJ, Leung WK, Go MY, et al. Cyclooxygenase-2 expression in Helicobacter pylori-associated premalignant and malignant gastric lesions. Am J Pathol 2000;157:729-35.

23 Mori N, Ueda A, Geleziunas R, et al. Induction of monocyte chemoattractant protein 1 by Helicobacter pylori involves NF-kB. Infect Immun 2001;69:1280-6.

24 Dufour JH, Dziejman M, Liu MT, et al. IFN-gamma-inducible protein 10 (IP-10; CXCL10)-deficient mice reveal a role for IP-10 in effector T cell generation and trafficking. J Immunol 2002;168:3195-204.

25 Sommer F, Faller G, Konturek $P$, et al. Antrum- and Corpus mucosa-infiltrating CD4+ lymphocytes in Helicobacter pylori gastritis display a Th1 phenotype. Infect Immun 1998;66:5543-6.

26 Gu L, Tseng S, Horner RM, et al. Control of TH2 polarization by the chemokine monocyte chemoattractant protein-1. Nature 2000;404:407-11.

27 Nakajima H, Kobayashi M, Pollard RB, et al. Monocyte chemoattractant protein-1 enhances HSV-induced encephalomyelitis by stimulating Th2 responses. J Leukoc Biol 2001;70:374-80.

28 Nakamura T, Lee RK, Nam SY, et al. Roles of IL-4 and IFN- $\gamma$ in stabilizing the helper cell type 1 and 2 phenotype. J Immunol 1997; 158:2648-53.

29 Iniguez MA, Martinez-Martinez S, Punzon C, et al. An essential role of the nuclear factor of activated T cells in the regulation of the expression of the cyclooxygenase- 2 gene in human T lymphocytes. J Biol Chem 2000;275:23627-35.

30 Tanaka K, Ogawa K, Sugamura K, et al. Cutting edge: Differential production of prostaglandin $\mathrm{D}_{2}$ by human helper T cell subsets. J Immunol 2000; 164:2277-80.

31 Gilroy DW, Colville-Nash PR, Willis D, et al. Inducible cyclooxygenase may have anti-inflammatory properties. Nat Med 1999:5:698-701.

32 Hans-Christian R, Loh EY, Ringler DJ, et al. Monocyte-chemoattractant protein 1 gene expression in intestinal epithelial cells and inflammatory bowel disease mucosa. Gastroenterology 1995;108:40-50.

33 Ross EL, D'Cruz, Morrow WJW. Localized monocyte chemotactic protein-1 production correlates with T cell infiltration of synovium in patients with Psoriatic arthritis. J Rheumatol 2000;27:2432-43. 